\title{
Noteworthy clinical case studies in cancer gene therapy: Tumor- targeted Rexin-G advances as an efficacious anti-cancer agent
}

\author{
ERLINDA M. GORDON and FREDERICK L. HALL \\ Epeius Biotechnologies Corporation, 475 Huntington Drive, San Marino, CA 91108, USA
}

Received February 26, 2010; Accepted March 30, 2010

DOI: 10.3892/ijo_00000619

\begin{abstract}
The advent of pathotropic (disease-seeking) targeting technology has ushered cancer gene therapy across the threshold of history, marking the beginning of a new epoch of medical praxis. For the first time, clinical oncologists can reach beyond the finest of catheters, beyond the reach of the most gifted surgeons, to the very fabric of metastatic disease in an effort to halt the progression and turn the tide of otherwise intractable cancers. The enabling molecular biotechnologies embodied in the leading tumor-targeted agent, Rexin-G, and its timely development as a safe and effective anti-cancer drug - from oncogene discovery and target validation, to molecular engineering of the core nanotechnologies, to the first clinical proofs-of principle, confirmatory trials, expanded access programs, and accelerated regulatory approvals - have been extensively documented in the medical literature. Therefore, this paper represents a final chapter, highlighting a series of noteworthy cases studies in the emergent field of targeted genetic medicine: case studies which, in and of themselves, reveal vital and important aspects of the molecular-genetic bio-pharmacology, advanced clinical protocols, refinement of patient monitoring, expanding treatment options, and strategic medical approaches to patient care that exemplify and thereby extend the established principles of pathotropic targeting and cancer gene therapy to a new generation of clinical practitioners.
\end{abstract}

\section{Introduction}

Rexin-G is a replication-incompetent, pathotropic (diseaseseeking), tumor matrix (collagen)-targeted retrovector encoding an N-terminal deletion mutant of the cyclin $G 1$ gene with potential antineoplastic activity [NCI Thesaurus, C49082] (1). Rexin-G is a highly engineered (2) and technologically sophisticated nanoparticle (3), which exhibits a physiological surveillance function that seeks-out diseased tissues, based

Correspondence to: Dr Erlinda M. Gordon, Epeius Biotechnologies Corporation, 475 Huntington Drive, San Marino, CA 91108, USA E-mail: egordon@epeiusbiotech.com

Key words: targeted genetic medicine, pathotropic targeting, metastatic cancer, cyclin G1, cell cycle, gene delivery, tumor response criteria on an intrinsic affinity to bind to newly exposed extracellular matrix proteins found throughout cancerous lesions. This physiological surveillance function was accomplished by the molecular engineering of a high-affinity collagen-binding motif, originally derived from von Willebrand factor (vWF), onto the retrovector's surface $(4,5)$. Exploiting the natural collagen-targeting mechanism of vWF permits efficient and selective delivery of the nano-sized retrovector to primary tumors and metastatic sites where angiogenesis and collagen matrix exposure characteristically occur $(6,7)$. The flagship of pathotropically targeted nanoparticles, designated Rexin-G, carries a cytocidal 'dominant negative' cyclin $G 1$ construct as the genetic payload $(8,9)$; a payload which has the ability to destroy or retard growth of tumor cells by disruption of cellular cyclin G1 activity, thus inducing apoptosis of tumor cells and the proliferative components of tumor-associated vasculature $(6,7,10)$.

In preclinical proof-of-concept studies, Rexin-G, given intravenously, has been shown to concentrate selectively in cancerous lesions and to attenuate tumor growth in human xenograft models of metastatic cancer $(6,7)$. In clinical studies, Rexin-G has demonstrated significant anti-tumor activity in a number of solid tumor types, including breast, colon, lung, skin, muscle and bone, as well as pancreas cancer $(11,12)$. Following on from initial Phase I safety studies (13) and adaptive Phase I/II studies (14,15), Rexin-G was granted Orphan Drug Status by the US FDA in 2008 for both soft tissue sarcoma and osteosarcoma, in addition to pancreatic cancer. Advanced Phase I/II clinical studies of Rexin-G for pancreatic cancer have shown that Rexin-G is well-tolerated, with an excellent safety/toxicity profile, and its repeated administration is associated with significant tumor regression and prolonged progression-free survival, with strong indications that Rexin-G monotherapy may improve overall survival as well (15). Following rigorous evaluations of the safety and efficacy of Rexin-G administered as stand-alone therapy for a broad spectrum of otherwise intractable cancers, Rexin-G was formally approved for the treatment of all chemotherapy-resistant solid tumors in the Republic of the Philippines, and has more recently been granted Fast Track status, as well as Orphan Drug priorities, by the US FDA.

As Rexin-G approaches the cusp of formal regulatory approvals in the USA for both pancreatic cancer and sarcomas, it is, perhaps, important to realize the potential clinical applications at hand vis-à-vis the number (and the time) of pivotal and confirmatory trials required to gain full international approvals for all solid tumors. And yet, that is the 
true clinical potential for a molecular-genetic agent with such exemplary safety and demonstrable broad spectrum anticancer activity-based on the inescapable crux of the cancer cell's universal replication machinery, that is, by virtue of its design (16). Indeed, recent studies of functional genomics using high-throughput screening methodologies have served to validate the cyclin $G 1$ locus as a critical gene target for the key tumor-suppressive, microRNA, miR-122 $(17,18)$, thereby linking the loss of this natural endogenous molecular regulation of the cyclin G1 locus to both the mechanisms of carcinogenesis (19) and the actual progression of metastatic disease (20). It now appears that, by inhibiting the executive oncogenic cyclin-G1 pathway in a highly effective manner, the Rexin-G retrovector serves to restore a natural tumorsuppressive function that is lost or disabled with the onset of many types of cancers. Moreover, the platform of pathotropic targeting which serves to deliver this broad spectrum 'designer gene' specifically to cancerous tissues, where it is needed most, is also fundamental to cancerous histopathology (16); and thus the targeting technology itself is broadly applicable. Consequently, it seems reasonable to propose that the objective tumor responses and significant survival benefits demonstrated by tumor-targeted Rexin-G in a set of precisely-defined clinical trials will eventually be extended to additional clinical indications and will involve an even greater number of medical centers in the near future.

Therefore, as a practicum for prospective clinical oncologists, and a summary of applicable experience gained in the crucible of clinical medicine, this article is presented as a series of ten noteworthy clinical case studies, each of which demonstrates, or teaches, a valuable aspect of the art of precision-targeted genetic medicine. From the very first Primer on Pathotropic Medicine, prepared by request for the bicentennial of the US FDA (21), we have respectfully offered our clinical experience and guiding principles to those who would venture forth in medical practice where no one has ventured before, advice we intentionally tinctured with due caution and resolve. Among these guiding principles was the axiom that the experience of each individual cancer patient treated within a diversity of clinical trials can, with an appropriate level of critical analysis, be of considerable benefit to the next. Once again, in the context of a practical discussion of these noteworthy cases, we offer our scientific and medical experiences and our foresight for scholarly review.

\section{Patients and methods}

Patient population. Case studies of eight patients with chemotherapy-resistant solid malignancies and two previously untreated patients (Case study nos. 06 and 10) are presented herein. The treatment protocols were reviewed and approved by the Institution's Review Board and the Biosafety Committee of Epeius Clinical Research Unit, San Marino, CA, USA. Written informed consent was obtained from each patient prior to treatment, in accordance with the Declaration of Helsinski protocols.

Treatment. The Rexin-G vector (11-15) is produced by transient co-transfection of three separate plasmids in $293 \mathrm{~T}$ cells (human kidney 293 cells transformed with the SV40 large $\mathrm{T}$ antigen) maintained as a fully validated master cell bank. The final clinical-grade product exhibits a viral titer of $5 \times 10^{9}$ colony forming units (cfu) per milliliter, a biologic potency of 50-70\% growth inhibitory activity in A375 melanoma cells, $<550$ bp residual DNA, no detectable E1A or SV40 large T antigen, and no detectable replication competent retrovirus (RCR). The clinical vector was stored in volumes of $23 \mathrm{ml}$ in $30 \mathrm{ml}$ vials in a $-80 \pm 10^{\circ} \mathrm{C}$ freezer marked for Biohazards. Preparation of the Rexin-G vector for patient administration consisted of rapid thawing of the vector in the vial in a $34^{\circ} \mathrm{C}$ waterbath. The vector was thawed 15-30 min prior to infusion into the patient, and then infused intravenously over 5-10 min at a rate of $\sim 4 \mathrm{ml} / \mathrm{min}$. All personnel who handled and disposed of the vector observed Biosafety Level 2 compliance, in accordance with the National Institutes of Health Guidelines for Research Involving Recombinant DNA molecules.

\section{Results}

\section{Ten noteworthy case studies}

i) Halting the progression of chemo-resistant cancers Case study Rx-01. 17-year-old white male with intractable metastatic osteosarcoma.

Clinical presentation. Chemo-resistant metastasis to heart, lungs, adrenal gland, and spine.

Treatment protocol. Rexin-G as stand-alone therapy; $1 \times 10^{11} \mathrm{cfu}$, given $2 \mathrm{x}$ a week.

Objective responses. $48 \%$ attenuation of metabolic activity determined by PET scan; along with extensive calcification and necrosis of the target lesions.

Case noteworthiness. Positive responses to Rexin-G by PET criteria prompted the FDA to 'green light' a confirmatory Phase II study.

Lessons from the practice of compassion. This 17 year-old white male, shown by radiography in Fig. 1A, was diagnosed with osteosarcoma of the right tibia in December 2003. He had received preoperative chemotherapy with cisplatin and adriamycin and high dose methotrexate followed by a limb salvage procedure. Post-operatively, he received courses of cisplatin and adriamycin (x2), and adriamycin and ifosfamide (x2), bringing the cumulative dose of adriamycin to $400 \mathrm{mg} / \mathrm{m}^{2}$. Chemotherapy was completed on February 2005. In March 2006, a follow-up CT-scan showed two left-sided pulmonary metastases which were removed by VATS thorascopic surgery. From June to November 2006, he received high dose methotrexate and ifosfamide, and then, underwent a thoracotomy in November 2006. From December 2006 to April 2007, his lung tumors grew in size and number from a single lung nodule measuring $1 \mathrm{~cm}$ to over 10 lung and pleuralbased nodules, with the largest lesion measuring $4.2 \mathrm{~cm}$. This rapid rate of disease progression was compounded by the lifethreatening location of the metastatic lesions, which involved both lungs, pericardium, and major vessels of the heart, with encroachment into the adrenal gland as well as the spine.

In April 2007, the patient received Rexin-G on a compassionate basis. The patient was given $1 \times 10^{11} \mathrm{cfu}$ Rexin-G intravenously twice a week for 4 weeks, followed by a 2-week rest period. A PET-CT scan obtained 1 week after completion 


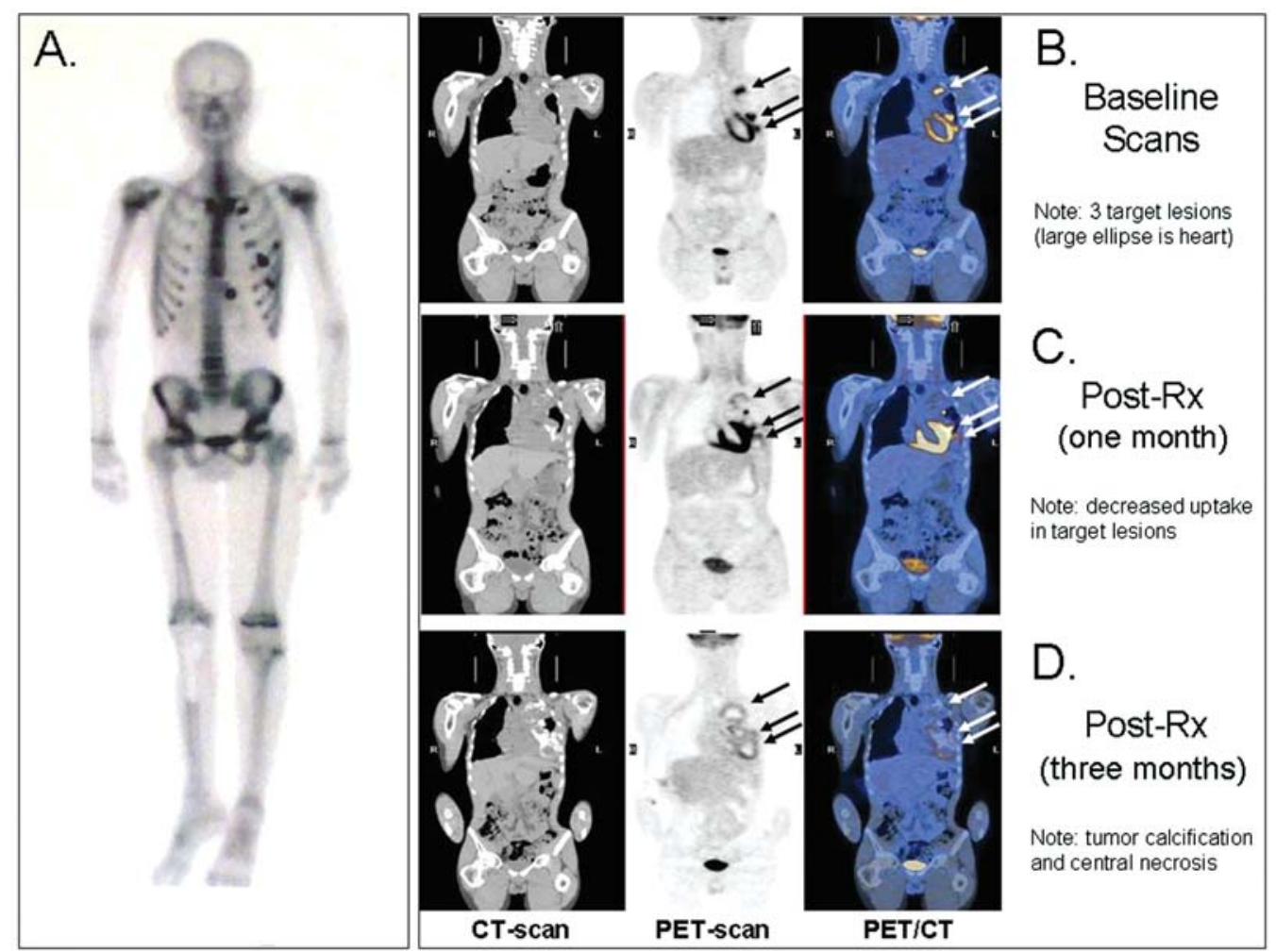

Figure 1. Case study (Rx-01) of intractable osteosarcoma, metastatic to heart, lungs, and adrenal gland. Radiologic imaging identifies the major metastatic sites (A), focusing on three pulmonary target lesions (arrows) which change dramatically from baseline (B), to 1 month (C) to 3 months (D) of Rexin-G treatment. Notably, the densities of these tumors change significantly, indicating reactive calcification and necrosis, while the PET scan adds mechanistic details, confirming the cessation of tumor metabolic activity.

of the first cycle showed a $28 \%$ increase in the sum of the target lesions, a $6 \%$ decrease in sum tumor density of target lesions, and a $33 \%$ reduction in the sum SUV max of 4 designated target lesions (Fig. 1B vs. C). He continued to receive Rexin-G for an additional 4 weeks. Remarkably, a PET-CT scan obtained 2 weeks after completion of the 2nd treatment cycle (Fig. 1D) showed no new lesions, a $48 \%$ reduction in the sum SUV max of the 4 major target lesions, and a 539\% increase in sum tumor density, indicating general calcification of the target lesions. Based, in part, on these positive tumor responses, the FDA approved a Phase II efficacy study of Rexin-G for metastatic osteosarcoma that is refractory to known therapies.

In this noteworthy case, Rexin-G treatment, even at relatively low doses, had a significant impact on this otherwise intractable cancer, not only halting the relentless progression of the disease, but preventing the occurrences of new lesions during the Rexin-G treatment period. In quantifying the objective tumor responses, a more comprehensive analysis of tumor response criteria was conducted - including PET criteria (metabolic activity), and CHOI criteria (tumor density), as well as RECIST (size only) - due to the tendency for osteocaroma lesions to calcify rather than shrink with the cessation of tumor cell proliferation. Based on the observed reduction of tumor growth and metastasis, in the context of such an obviously poor initial prognosis, the US FDA gave a 'green light' for ongoing Phase I/II studies to proceed to a confirmatory Phase II trial, specifically for osteosarcoma, while the performance status of this Compassionate Use patient remained well enough for him to complete the requirements to achieve Eagle Scout distinction.

Case study Rx-02. 38-year-old black female with intractable metastatic osteosarcoma.

Clinical presentation. Chemo-resistant osteosarcoma with tumor metastasis to the lungs.

Treatment protocols. Rexin-G as stand-alone therapy; $1-2 \times 10^{11} \mathrm{cfu}$, given $3 \mathrm{x}$ a week.

Objective responses. Attenuation of tumor metabolic activity, determined by PET criteria, sufficed to justify surgical resection.

Case noteworthiness. Approved dose escalation enables tumor control and a subsequent surgical remission; adjuvant Rexin-G therapy sustains remission for $>2$ years.

Effectuating a surgical remission. The aforementioned Phase II efficacy study of Rexin-G for the treatment of chemo-resistant osteosarcoma brought forth an opportunity for the demonstrated anticancer activity of Rexin-G to serve as neoadjuvant therapy, thus setting the stage for a potentially curative surgery. In this case, a 38 year-old female was diagnosed in September 1995 to have localized osteosarcoma of the left fibula. She underwent a limb salvage procedure in January 1996, where the neoadjuvant/adjuvant therapy consisted of methotrexate, ifosfamide, cisplan and adriamycin. Over the years, she developed multiple pulmonary metastases, requiring surgical resection of lung tumors, followed by re-institution of methotrexate, ifosfamide, adriamycin and cisplatin, plus 
interferon. In January 2008, she presented with chemo-resistant lung metastasis and was enrolled in the Phase II study using Rexin-G for osteosarcoma - which consisted of Rexin-G i.v. at a dose of $1-2 \times 10^{11} \mathrm{cfu}$, administered three times a week for 4 weeks, with a 2 -week rest period.

Having failed a number of aggressive chemotherapeutic regimens, and following previous rounds of surgical excisions, the cancer had recurred, presenting as a single lung metastasis. Repeated intravenous infusions of tumor-targeted Rexin-G included an intra-patient dose escalation in this case, which was approved across-the-board by the US FDA, once adequate safety had been determined in ongoing clinical trials. Treatment with Rexin-G had a significant impact on the histology of the tumor, which upon surgical resection, was shown to have undergone cystic conversion of the one metastatic target lesion and ossification of an occult lesion, i.e., not seen by PET-CT scan (Fig. 2). Thus, the patient received three treatment cycles followed by surgical resection of the residual lung tumors, and then 5 more cycles of Rexin-G postoperatively. To date, two years later, she enjoys a sustained remission with no evidence of disease.

Case study Rx-03. 36-year-old white male with intractable Ewing's sarcoma.

Clinical presentation. Chemotherapy-IGFR-therapyresistant metastasis to the lung.

Treatment protocols. Rexin-G as stand-alone therapy; $2 \times 10^{11}$ cfu infusions daily, $5 \mathrm{x}$ a week.

Objective responses. Attenuation of metabolic activity by PET; stabilization of tumor growth.

Case noteworthiness. Corroborative PET radiologic studies refine tumor response analysis.

Refinement of RECIST tumor response criteria. Ewing's sarcoma is a relatively rare malignancy of the bone and soft tissues, which is generally treated aggressively with multidrug chemotherapy, in addition to local disease control with surgery and/or radiation. In cases where progression to metastatic disease is apparent and the patient becomes refractory to standard therapies, the prognosis is exceedingly poor. In this case, a 36 year-old male was diagnosed with Ewing's sarcoma which was metastatic to lung and liver in July 2004. His multidrug chemotherapy regimens consisted of doxorubicin, dacarbazine, and ifosfamide, in addition to radiotherapy and surgical resection. After failing standard therapy, he was enrolled in a Phase I clinical study of a monoclonal antibody, i.e., the RG1507 antibody by Hoffman-LaRoche, directed against the IGF receptor. The patient responded transiently to insulin-like growth factor-1 receptor (IGF-1R) therapy, which became ineffective over time (additional note, in December of 2009, Roche/Genentech announced their decision to discontinue the clinical development of RG1507. Likewise, Pfizer suddenly suspended its Phase III figitumumab IGF-1R trial after a critical futility analysis).

After failing this trial, the heavily pretreated patient received Rexin-G as stand-alone salvage therapy administered 5 days a week in an advanced induction regimen: Rexin-G i.v., given two times each day at a dose of $2 \times 10^{11}$ cfu per infusion. A subsequent PET/CT scan showed the persistence of large tumor masses in the lungs, yet there was a marked attenuation of metabolic activity in two of the largest lung nodules, as determined by an analysis of the composite of radiologic images. As seen in Fig. 3, the location and amount of the progressive metastatic disease in the lungs was considerable at this point of Rexin-G salvage therapy; however, the anti-tumor activity of the 2x daily Rexin- $G$ infusions became more evident upon careful analysis of the PET/CT scans. An overlay of the CT scan, which simply shows the size of the major pulmonary lesions, with the PET scan (PET/CT scan), which reveal the actual metabolic activity within these tumors, uncovered the true extent of the impact on tumor growth, as two of the three of the major target lesions showed significantly reduced metabolic activity (Fig. 3A), while the third, a metabolically active lesion, exhibited a discernibly necrotic center. Moreover, similar comparative scans of the spinal musculature of the lumbar region (Fig. 3B) reveal troublesome evidence of tumor metastases by PET/CT that was not recorded by CT scan alone. These noteworthy observations indicate that the understanding gained by CT scans alone, is of a very meager kind, and suggest that a refinement of tumor response criteria to include evaluation of tumor metabolic activity be considered when it comes to precision targeted molecular therapies.

After three Rexin-G treatment cycles, the patient, by responding favorably to Rexin-G monotherapy, qualified for enrollment in the new GeneVieve protocol, consisting of Rexin-G plus Reximmune-C [i.e., tumor-targeted GM-CSF vaccine (3)] in an effort to prompt localized immune responses within the residual tumors, which might, in principle, lead to additional anti-tumor activity and long lasting anti-tumor immunity.

Case study Rx-04. 74-year-old white female with intractable metastatic breast cancer.

Clinical presentation. Chemo- and hormone-resistant cancer metastases to lymph nodes and chest wall.

Treatment protocols. Rexin-G as stand-alone therapy; $2 \times 10^{11}$ cfu given $3 \mathrm{x}$ a week.

Objective responses. Tumor shrinkage enabling surgical resection of a residual tumor nodule.

Case noteworthiness. Tumor histology confirms more significant cytological efficacy, including favorable immune responses; survival >3-years following treatment.

Recruitment of immune cells into the tumor microenvironment. This noteworthy case is a 74-year-old white female with recurrent ductal carcinoma of the breast, metastatic to axillary lymph nodes and tissues of the chest wall. She was diagnosed in September 2001 to have infiltrating ductal carcinoma of breast, T3N2 stage, for which she underwent a right mastectomy in September 2001, received doxorubicin and cyclophosphamide, radiation to the chest wall, followed by docetaxel, and then tamoxifen which was initiated in October, 2002. The breast cancer was determined to be ER-positive, and questionable for HER-2/neu positivity. The patient remained on tamoxifen until November, 2006, when she recurred in the chest wall, supraclavicular, axillary, and mediastinal lymph nodes, and possibly bone. She was entered in a clinical trial using Faslodex from November 30, 2006 to January 25, 2007. The patient responded initially, but there was residual 

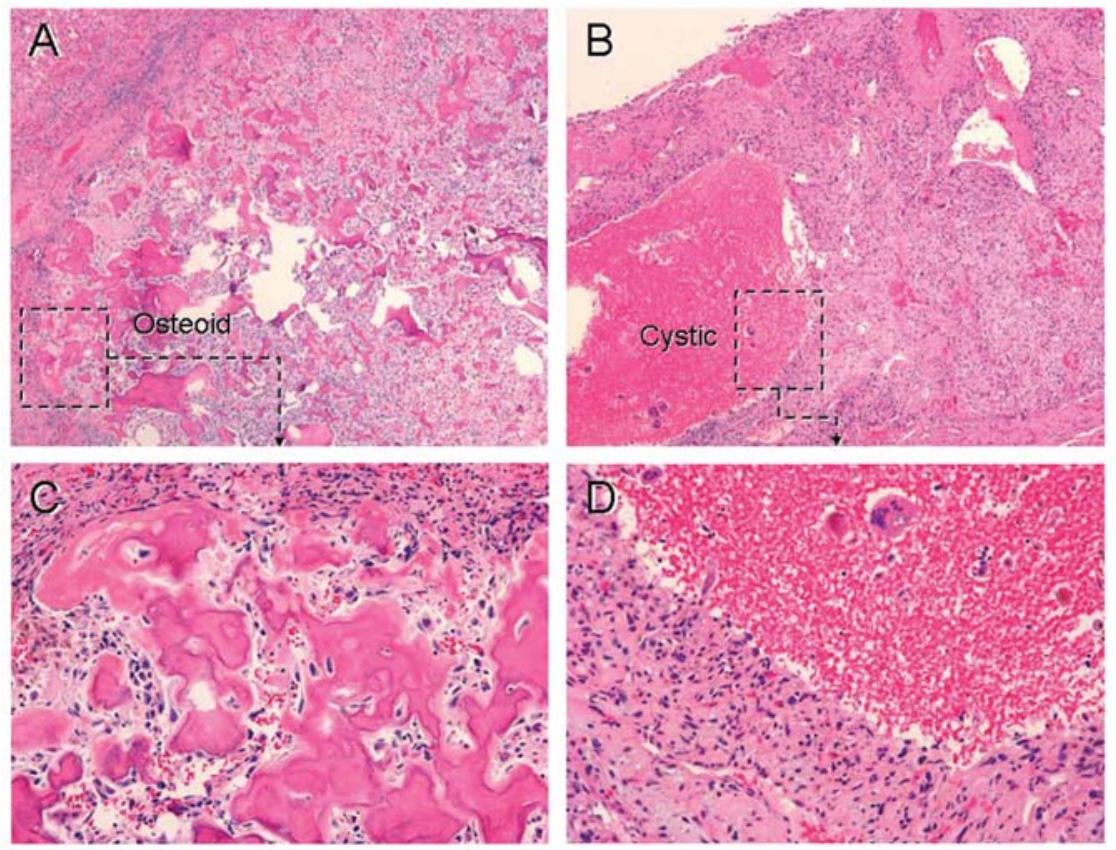

Figure 2. Case study (Rx-02) of intractable metastatic osteosarcoma wherein halting progression and stabilization of disease by Rexin-G, acting here as neoadjuvant and adjuvant therapy, enabled a surgical remission gained by the excision of two residual tumor nodules. Histological examination of the excised tumors demonstrated clear objective responses, confirming calcification (A and $\mathrm{C}$ at higher magnification) in one lesion, and cystic conversion and necrosis (B and D at higher magnification) of the second lesion following Rexin-G treatment.

\section{A. Chest Region: multiple non-calcified pulmonary lesions in both lung fields}
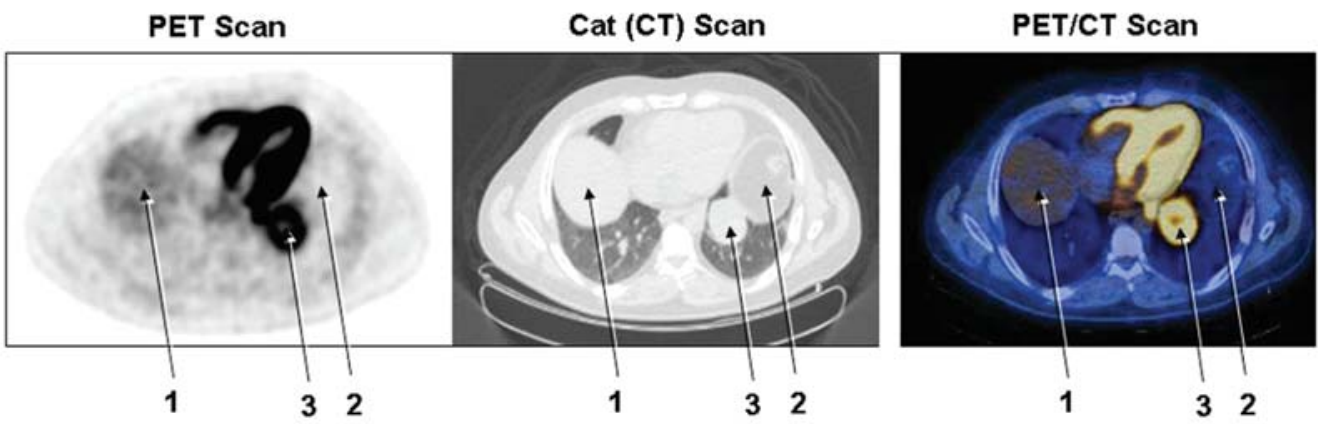

B. Lumbar Region: diffuse uptake (lesions) in soft tissues with foci in muscles

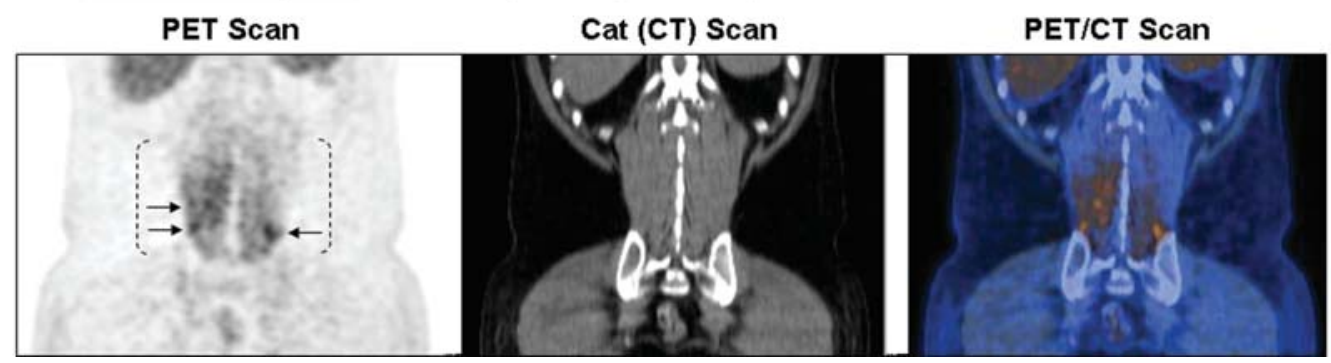

Figure 3. Case study (Rx-03) of intractable Ewing's sarcoma, metastatic to the lungs and spine. A comparison of the PET scans with the CT scans of three large target lesions in the chest region (A) reveals a problematic disparity in evaluating objective clinical responses in tumor size versus tumor metabolism following Rexin-G treatment. Likewise, the diffuse metastastic tumor infiltration in the lumbar region (B), which was detected by PET scan but not CT scan, further suggests that clinical understanding based on tumor size alone is of a very meager kind.

therapy-resistant disease that was confirmed by repeat CT scans on February 8, 2006.

In this case of chemotherapy-resistant, hormone-resistant breast cancer, the recurrent disease was manifested in both lymph nodes and the anterior chest wall. Repeated infusions of Rexin-G, $1 \times 10^{11}$ cfu given three times a week for 3 weeks, resulted in regression of the chest wall tumor and axillary lymph nodes, enabling surgical resection of the solitary residual 
tumor. As shown in Fig. 4, the residual tumor was far from a flagrant proliferative tumor, appearing largely as a fibrotic mass (blue-staining material on Masson's trichrome stain) with scant but discernable apoptotic tumor cells accompanied by significant tumor infiltrating lymphocytes (TILs). Further characterization of the complement of TILs by specific immunocytochemical staining identified a significant proportion to be $\mathrm{CD}^{+}$killer T-cells, which are generally associated with a more favorable prognosis, a more favorable prognosis, that is affirmed by the continued survival of this patient, who is still alive more than three years after Rexin-G treatment.

Case study Rx-05. 61-year-old Asian female with intractable metastatic ovarian cancer.

Clinical presentation. Chemotherapy-resistant cancer with metastasis to cerebrum and brain stem.

Treatment protocols. Rexin-G as stand-alone therapy; $2 \times 10^{11}$ cfu given 5 days a week.

Objective responses. Regression of metastatic brain lesions in frontal lobe and cerebellum.

Case noteworthiness. First clinical demonstration of tumor control across the blood-brain barrier.

Rexin-G nanoparticles cross blood-brain barrier. This 60-yearold patient was diagnosed to have adenocarcinoma of the left ovary, metastatic to omentum in May, 2006. She underwent a total abdominal hysterectomy with bilateral salphingo-oophorectomy and received 6 cycles of paclitaxel and carboplatin with radiotherapy to the left pelvis. In November, 2009, she developed metastases to the left frontal lobe and right cerebellum, associated with severe depression and lethargy. She then received relatively intensive doses of Rexin-G monotherapy i.v. at $2 \times 10^{11}$ cfu per dose, given 5 days a week for 8 weeks. This intensive Rexin-G treatment resulted in substantial improvements in her depression and cognition, concomitant with regression of the cerebral and cerebellar metastatic foci.

This is not the first demonstration of Rexin-G singleagent efficacy seen in ovarian cancer, for objective tumor responses by RECIST have been recorded previously (data not shown). This case is particularly noteworthy as one of the first documented demonstrations of clinical efficacy, achieved by simple intravenous infusion that reached across the physiologically daunting blood-brain barrier. Whether the transport of these therapeutic doses of tumor-targeted Rexin-G nanopartilces across the blood-brain barrier and/or the choroid plexus is mediated by the retrovector surface envelope proteins (22) or by some mechanism(s) of capillary permeability related to the disease histopathology (23), it is clear that Rexin-G exhibits sufficient penetrance and therapeutic mass action concentrated at the level of the individual brain tumors to cause the anatomical regression of these lesions.

Case study Rx-06. 91-year-old white Catholic priest with metastatic prostate cancer.

Clinical presentation. Primary tumor with extensive painful bone metastases.

Treatment protocols. Rexin-G as stand-alone therapy; $2 \times 10^{11} \mathrm{cfu}$, given $3 \times$ per week.
Objective responses. Eradication of the primary tumor and non-progression of bone metastases, resulting in progressive relief from bone pain and increased mobility.

Case noteworthiness. First clinical demonstration of Rexin-G single-agent efficacy in advanced metastatic prostate cancer.

All things are possible to him that believeth. This 91-year-old priest was diagnosed to have metastatic prostate cancer in April, 2009. He presented with a primary prostate gland malignancy with involvement of the urinary bladder floor, seminal vesicles, and obstructive uropathy, resulting in bilateral hydronephrosis; also evident was a high PSA level and extensive skeletal metastasis (skull, scapulae, sternum, vertebrae, ribs, pelvis, iliac wings, ischium, pubic bones, and femur) associated with debilitating bone pain to the extent that the patient was bedridden with ensuing decubitus ulcers. Due to the advanced age of this patient, first-line treatment with toxic chemotherapies and/or radiation therapy was precluded. Instead, the patient received Rexin-G i.v., $2 \times 10^{11} \mathrm{cfu}$ per dose given three times a week for 8 weeks. Among the first distressing symptoms to abate was the severity of the bone pain followed by progressive relief from the sequelae of hydronephrosis. Follow-up abdominal sonogram, CT scans, and bone scans showed a normal prostate gland and kidneys, with non-progression of the bone metastases; in addition to subjective relief from pain, there was a significant reduction in serum PSA levels.

The elderly priest was eventually able to walk again with the aid of a walker, to participate in daily mass, and to resume his distinguished lectures to resident seminarians. In addition to the noteworthy demonstration of Rexin-G safety and efficacy, observed here at the edge of hospice care, are: i) the social and religious acceptability of cancer gene therapy seen advancing as something wholesome and beneficial, ii) the medical acceptability whereby a clinical oncologist is sufficiently confident to prescribe Rexin-G as first-line therapy, and iii) a reminder that the observed broad-spectrum anti-cancer activity of Rexin-G includes yet another type of solid tumor.

\section{ii) Turning the tide of intractability in pancreatic cancer} Case study Rx-07. 54-year-old Asian female with intractable metastatic pancreas cancer.

Clinical presentation. Chemo-resistant unresectable pancreas cancer metastatic to liver, abdominal lymph nodes, and lung.

Treatment protocols. Rexin-G given as stand-alone therapy; $2 \times 10^{11} \mathrm{cfu}$, given $3 \mathrm{x}$ a week.

Objective responses. Resolution of primary tumor and regression of liver metastasis by CT scan.

Case noteworthiness. Resolution of primary tumor after only 4 weeks of Rexin-G treatment.

Eradication of the primary tumor. This 60 -year-old female was diagnosed in January, 2009 to have pancreatic adenocarcinoma with metastasis to the mesentery, liver, and lungs. The patient underwent a biliary bypass and was treated with standard chemotherapy, gemcitabine $1000 \mathrm{mg} / \mathrm{m}^{2}$ for 4 weeks, which soon failed and resulted in progression of the disease. In April, 2009, she started treatment with Rexin-G i.v. at 

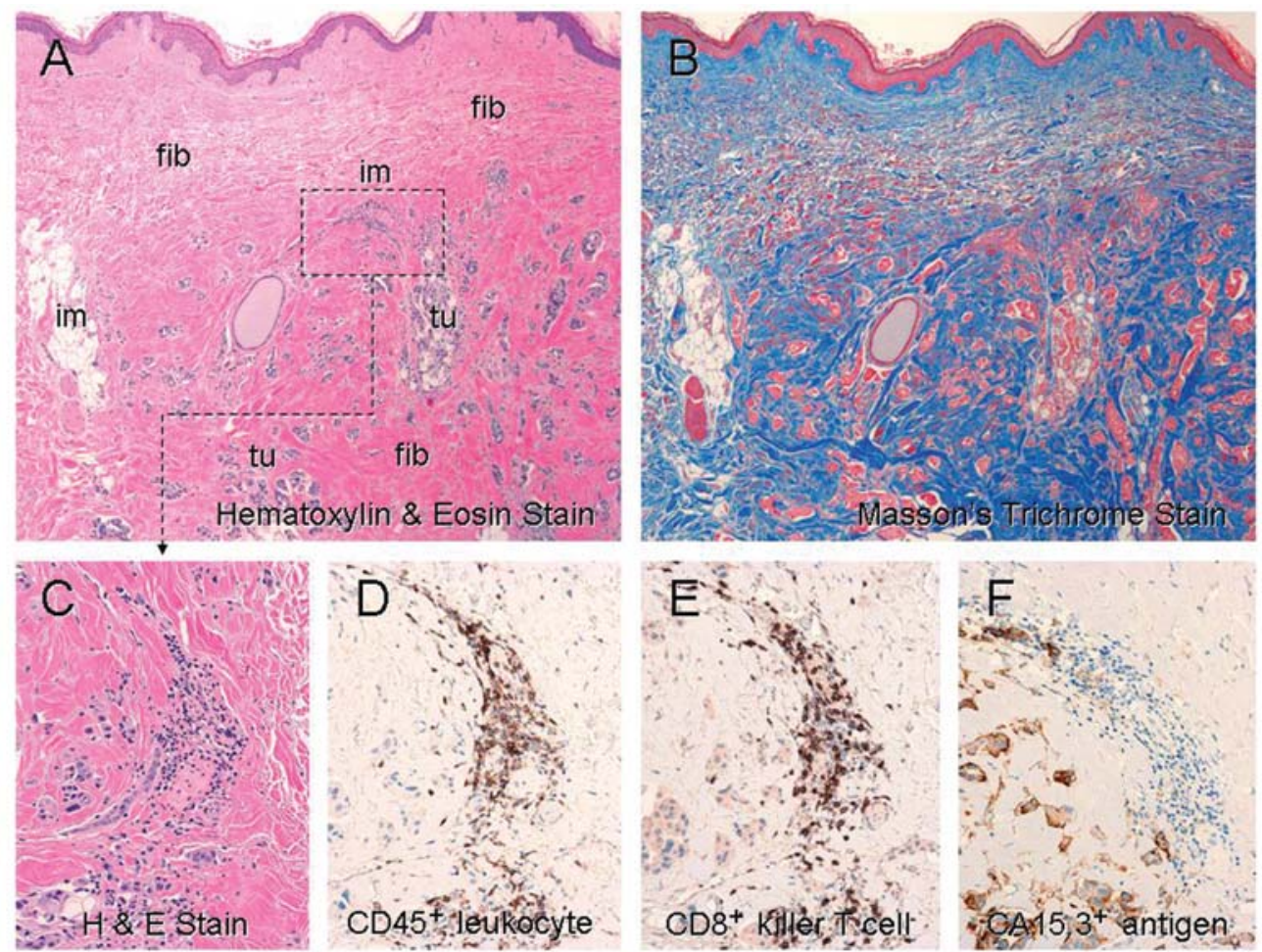

Figure 4. Case study (Rx-04) of intractable metastatic breast cancer reveals histological aspects of tumor destruction, reparative fibrosis, and reactive immune cell infiltration, now-classical hallmarks of Rexin-G action. In this excised tumor nodule, a scant number of tumor cells (tu) can be seen in the context of extensive fibrosis (fib) accompanied by a significant immune response (im) following Rexin-G treatment (A, H\&E staining; B, trichrome stain for extracellular matrix proteins). Far from a flagrant tumor, the remaining nests of degenerative tumor cells (marked in F) appear to be infiltrated and 'recognized' by the patient's immune cells (C, H\&E; D, LCA immunostaining), including killer T-cells (E).
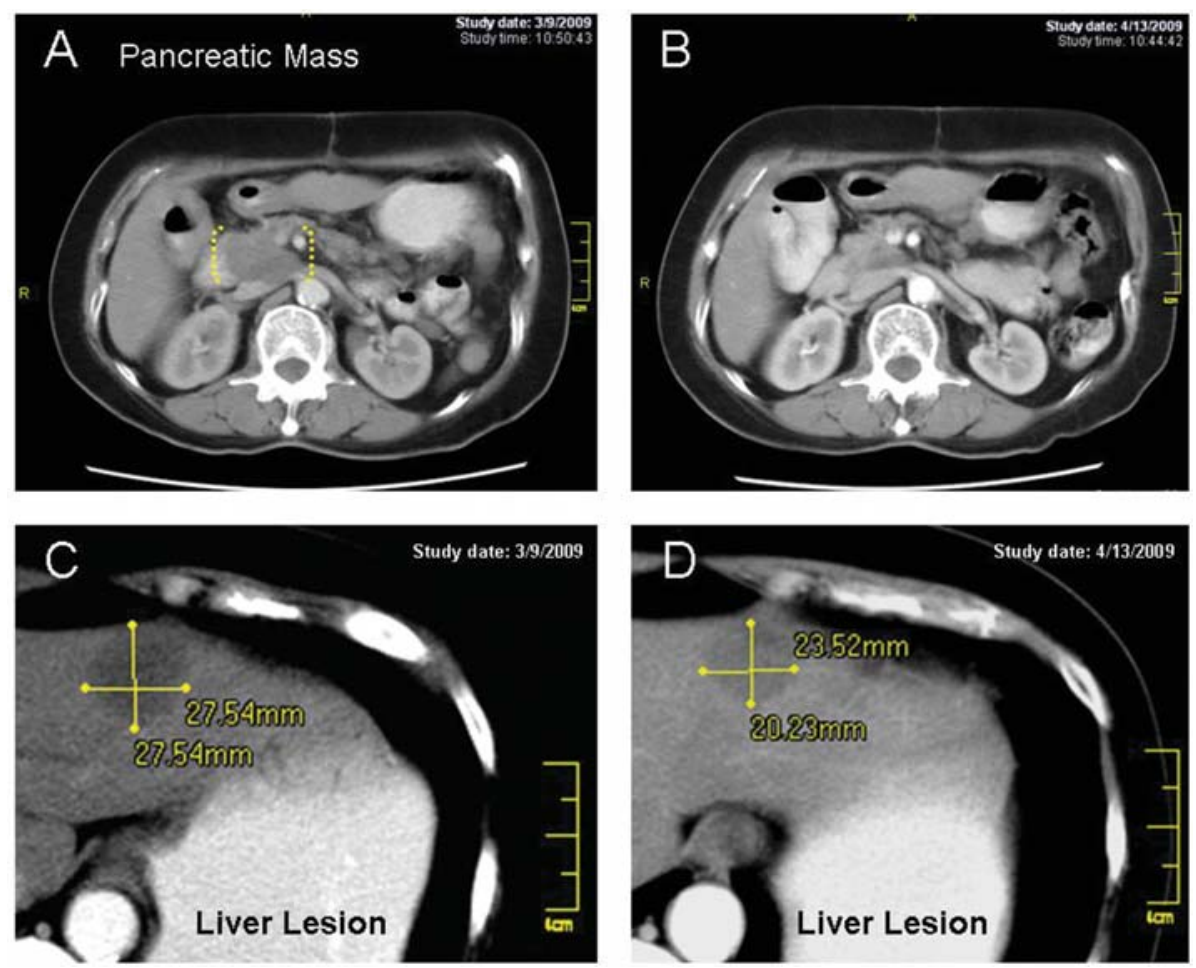

Figure 5. Case study (Rx-07) of intractable metastatic pancreatic cancer, wherein the patient received Rexin-G as second-line therapy treatment shortly after failing standard first line therapy; thus demonstrating the clinical benefit of gaining effective tumor control at a relatively early stage of disease progression. Complete regression of the primary pancreatic tumor (A versus B) is demonstrated along with both size (RECIST) and density (CHOI) changes in a metastatic liver lesion (C versus D); resulting in the stabilization of disease, prevention of new lesions, and enhancement of treatment options.

$2 \times 10^{11} \mathrm{cfu}$ per dose, given three times a week for 4 weeks. Follow-up CT scan at the end of 4 weeks showed complete regression of the primary tumor and reduction in the size of the liver metastasis (target lesion). As seen in Fig. 5, there 
was a prompt and discernable change in tissue density (CHOI criteria), as well as tumor size (RECIST) following Rexin-G treatment. After one notable cycle of Rexin-G administered as second-line therapy, this favorably-responding patient was enrolled in the GeneVieve Protocol, consisting of Rexin-G plus Reximmune-C (targeted GM-CSF) personalized vaccine therapy. She has presently completed the 6-month treatment with Rexin-G + Reximmune-C without event, is alive and well with no new lesions and confirmed stable residual disease, and is currently undergoing treatment with Rexin-G as maintenance therapy for another 6 months.

Case study Rx-08. 73-year-old white female with intractable metastatic pancreas cancer.

Clinical presentation. Chemo-resistant with metastasis to liver and abdominal lymph nodes.

Treatment protocols. Rexin-G as stand-alone therapy; $3 \mathrm{x}$ $10^{11} \mathrm{cfu}$, given $3 \mathrm{x}$ a week.

Objective responses. Complete clinical remission gained by maintaining treatment for 9 months.

Case noteworthiness. First demonstration of Rexin-Ginduced clinical remission in a patient presenting with metastatic chemotherapy-resistant pancreatic cancer.

Induction of a complete response and sustained clinical remission by holding the course. This 73-year-old female was diagnosed to have adenocarcinoma of pancreas in June, 2006. The patient underwent a Whipple's procedure in July, 2008 and received adjuvant therapy with 5-FU from September, 2006 to October, 2006, followed by gemcitabine from November, 2006 to February, 2007. She suffered tumor recurrence in the liver and abdominal lymph nodes in October, 2008, and was subsequently enrolled in a Phase I/II study of Rexin-G for gemcitabine-resistant pancreas cancer. She received Rexin-G i.v., at $3 \times 10^{11}$ cfu per infusion three times a week for 4 weeks followed by a 2 -week rest period (comprising one treatment cycle). There were no new lesions during 6 months of Rexin-G treatment, indicating stable disease (SD, Fig. 6A); however, there was some concern that one of the remaining liver lesions appeared to be slightly larger (by RECIST), which could be suggestive of progressive disease (PD). A further, more comprehensive analysis of objective tumor responses, including the progressive reduction in size of the target lymph node lesion (Fig. 6B) and a sustained drop in CA19.9 levels to near-normal levels (Fig. 6C) encouraged the Principal Investigator to hold-thecourse of Rexin-G treatment - resulting, ultimately, in a complete clinical remission (CR, Fig. 6A), as the remaining liver lesion was promptly resolved.

The observed absence of new lesions during repeated cycles of Rexin-G treatment, along with the achievement of stable disease (SD) represent significant clinical benefits, which should not be underestimated, in light of the predictable behavior of pancreatic cancer and the molecular mechanisms of action of Rexin-G. The continued treatment of this noteworthy pancreatic cancer patient, who was declared to be in clinical remission after 9 months of Rexin-G treatment, serves as a reminder that the eradication of metastatic liver lesions may occur promptly via apoptosis and anti-angiogenesis, or resolve gradually with the onset of fibrosis and tumor infiltrating lymphocytes (10), in which case it is of considerable benefit
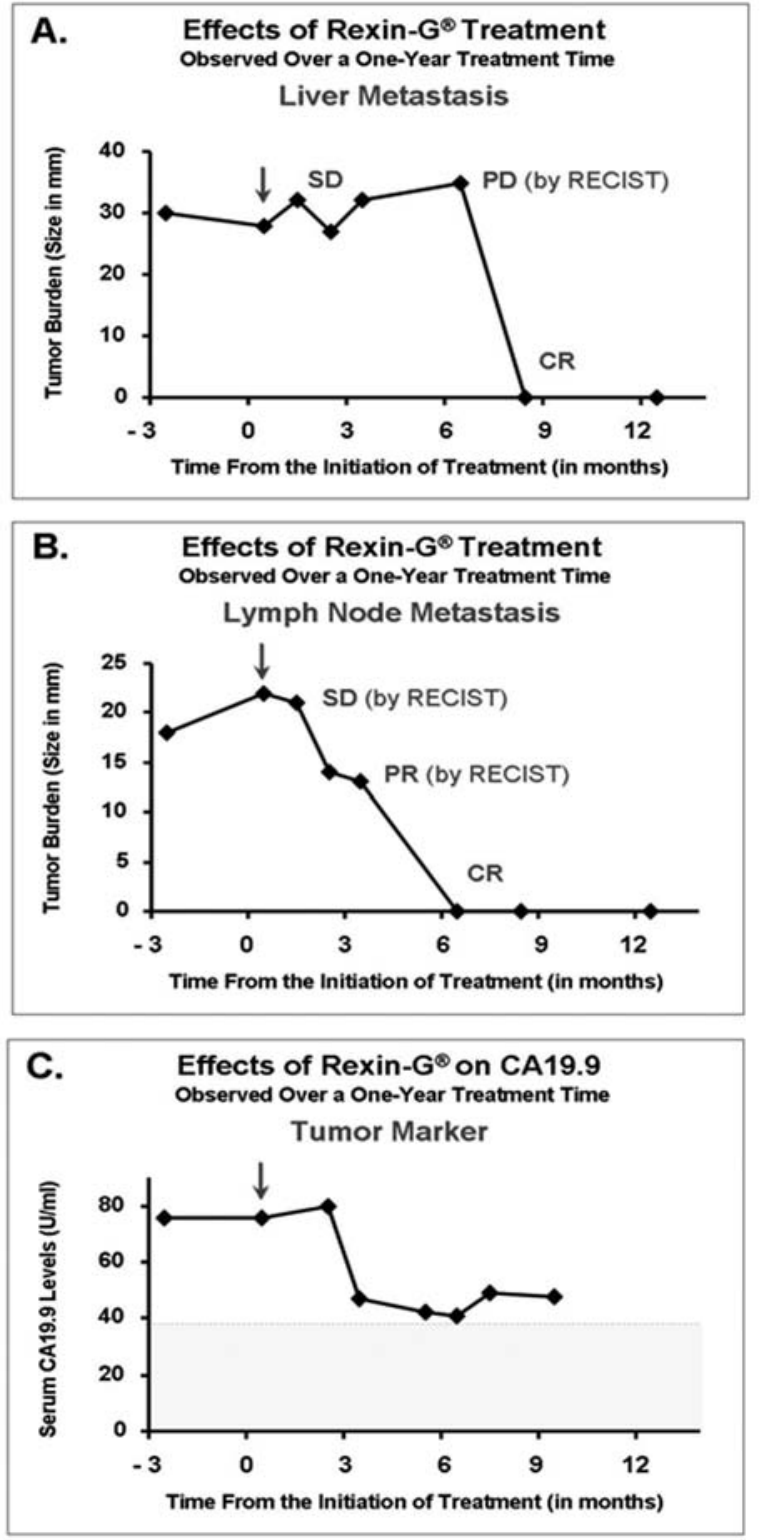

Figure 6. Case study (Rx-08) of recurrent chemotherapy-resistant pancreas cancer with metastasis to the liver and abdominal lymph nodes, documenting a complete clinical remission gained by continued treatment with Rexin-G as stand alone therapy. Graphic analysis of radiological images of tumor burden in the liver (A, Y-axis) obtained during course of Rexin-G treatment (X-axis) demonstrated a halting of progression with stable disease (SD) and no new lesions; however, a slight rise in the size a liver lesion (determined solely by RECIST criteria) 'appeared' to indicate progressive disease (PD). Fortunately, a more comprehensive analysis of the eradication of tumor burden in the lymph nodes (B), including the levels of the CA19.9 tumor marker $(\mathrm{C})$ which had dropped toward baseline, encouraged the oncologist to hold-the-course of the targeted therapy, thereby maintaining the conditions that led to a complete tumor response (CR) within the following month. The importance of holding the course of Rexin-G treatment, in the absence of systemic toxicity, in the absence of any new lesions and/or verifiable disease progression, is evident by the resulting sustained clinical remission.

to continue to hold-the-course of Rexin-G treatment. At this time, this noteworthy pancreas cancer patient continues to enjoy a sustained remission for greater than 16 months from the initiation of Rexin-G treatment.

Case study Rx-09. 50-year-old white female with intractable metastatic pancreas cancer. 

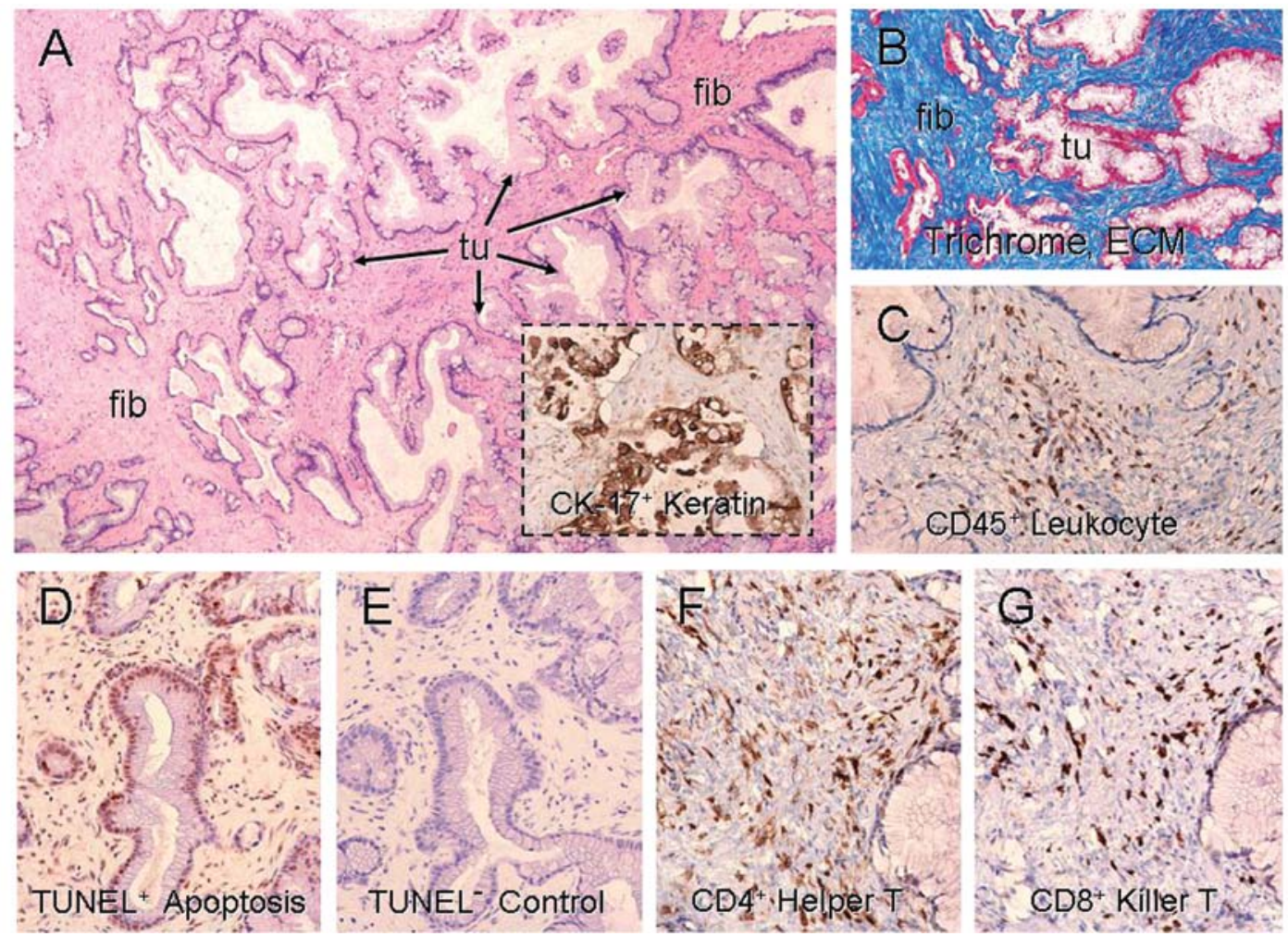

Figure 7. Case study (Rx-09) of intractable metastatic pancreas cancer, wherein the surgical excision of a residual tumor from the liver provides important insights into the molecular mechanisms-of-action of Rexin-G, as well as a sustained clinical remission. Histological examination of the excised liver nodule (A) demonstrates the limitations of simple RECIST measurements, revealing epithelioid tumor cells (tu) in various stages of degeneration (insert) that are surrounded by a significant amount of reparative fibrosis (B, ECM stains blue) and immune cell infiltration (C, leukocytes), including both helper T-cells (F) and killer T-cells (G). Most noteworthy is the direct anti-tumor action of Rexin-G, which is evidenced by the stunning amounts of apoptosis (active cell death) seen in the columnar/ductal arrays of tumor cells (D, TUNEL stain; E, control); for the curative surgical excision of this nodule followed Rexin-G treatment, as neoadjuvant therapy.

Clinical presentation. Chemotherapy-resistant post Whipple's recurrence, metastasis to liver.

Treatment protocols. Rexin-G as stand-alone therapy; $4 \times 10^{11}$ cfu given $3 \mathrm{x}$ a week.

Objective responses. Tumor progression is halted with disappearance of liver metastasis; a single residual tumor is excised after 6 months of Rexin-G therapy.

Case noteworthiness. Surgical remission is enabled by Rexin-G treatment, providing direct histological evidence of the molecular mechanisms-of-action.

'Veni, vidi, vici' ... quoth the nanoparticle. This 50 year-old female was diagnosed in August, 2007 to have adenocarcinoma of the pancreas. The patient underwent a Whipple's procedure followed by a course of adjuvant chemotherapy consisting of gemcitabine and capecitabine from November, 2007 to March, 2008. In February, 2009, follow-up CT scan showed several foci of liver metastasis. She was then entered into a Phase I/II study of Rexin-G for gemcitabine-resistant pancreas cancer in March, 2009, where she received 4 cycles of Rexin-G i.v. at $4 \times 10^{11} \mathrm{cfu}$ per dose administered three times a week, which resulted in the stabilization of disease progression, the prevention of new lesions and the eradication of one of two metastatic liver nodules (target lesions). The prevention of new lesions from occurring during the Rexin-G treatment period enabled the Principle
Investigator to recommend a surgical resection of the one solitary residual tumor; which was promptly excised and embedded for histological examination.

The timely treatment of this patient with Rexin-G, as neoadjuvant, immediately prior to the surgical procedure, enabled an opportunistic examination of Rexin-G in action within the metastatic lesion. As shown in Fig. 7A, a significant proportion of the volume of this Rexin-G pretreated tumor is composed of fibrosis and extracellular matrix proteins (Fig. 7B), while the remainder of the residual tumor appears to be a rather slow growing and relatively pseudo-differentiated array of columnar/ductal structures in various stages of degeneration. This observation confirms the assertion that the objective response to treatment may be grossly underestimated by mere RECIST measurements. More remarkably, Rexin-G appears to have induced massive amounts of apoptosis of the remaining cancer cells (see TUNEL stain in Fig. 7D), as well as visible karyorrhexis, which is evident all along the borders of the pseudo-glandular structures. While the patient's local immune response is far from robust, with sporadic infiltration of $\mathrm{CD}_{4} 5^{+}$leukocytes observed within the lesion (Fig. 7C), the cellular infiltrate consisted majorly of $\mathrm{CD}^{+}$helper T-cells (Fig. 7F) and CD8 $8^{+}$killer T-cells (Fig. 7G).

In addition to controlling the growth and spread of metastatic disease in Stage IV pancreatic cancer using Rexin-G as stand-alone therapy, this case is particularly noteworthy: 
for Rexin-G, by acting as an effective adjuvant therapy, enabled a definitive surgical remission from this deadly form of cancer, which is important for both clinical and surgical oncologists to consider. Post-operatively, the patient resumed Rexin-G treatment, after healing from the procedure, and continues to enjoy sustained clinical remission for $>11$ months after treatment initiation.

Case study Rx-10. 47-year-old white male with intractable metastatic pancreas cancer.

Clinical presentation. Primary pancreatic mass with extensive liver and abdominal lymph node metastases.

Treatment protocols. Rexin-G first-line with gemcitabine; Rexin-G, 2-3x10 11 cfu, given 5 days a week; plus gemcitabine $1000 \mathrm{mg} / \mathrm{m}^{2}$, given weekly x 7 weeks.

Objective responses. Prompt regression of primary tumor with $40 \%$ reduction in CA19.9 level.

Case noteworthiness. Landmark first-line combination therapy with Rexin-G plus gemcitabine, devised to potentiate tumor responses to the oncolytic antimetabolite.

Questing for synergism. Presenting with symptoms of fever and jaundice, this 48-year-old white male was diagnosed to have adenocarcinoma of the pancreas with an extensive metastatic tumor burden involving the liver and abdominal lymph nodes in November of 2009. While a Whipple's procedure was precluded by the presence of the metastatic disease, a biliary bypass was performed with choledocoduodenal anastomosis and cholecystectomy. Responding to an urgent request for compassionate use of Rexin- $G$ as firstline therapy, and following all the qualifications and ramifications of international regulatory approvals, the patient was treated with a combination of Rexin-G-given i.v. at $2 \times 10^{11} \mathrm{cfu}$ per dose, 5 days a week-plus gemcitabine administered at a weekly dose of $1000 \mathrm{mg} / \mathrm{m}^{2}$ for a total 7 weeks. Following the initial course of this first-line combination therapy, a follow-up MRI showed significant regression of the pancreatic mass and a general stabilization of the liver metastases and abdominal lymphadenopathy. These radiologic indications of tumor control were accompanied by a $30 \%$ reduction in the level of the tumor marker CA19.9, which is additionally noteworthy in light of studies suggesting that a timely decline in CA19.9 compares favorably with objective radiological responses as a strong indicator of time-to-progression, as well as overall survival, and may even serve as a surrogate end-point $(24,25)$.

The gemcitabine was discontinued for a period of 2 weeks, due to a progressive elevation in liver enzyme levels (i.e., LFT elevation), attributable to known gemcitabine toxicity, in accordance with standard dose/treatment modification protocols; while the Rexin-G infusions were continued during this extended rest period. Notably, the liver function tests promptly normalized while the CA19.9 continued to fall to $40 \%$ of the initial values. With the relative safety of the combined therapy established, the dose of Rexin-G was raised to $3 \times 10^{11}$ cfu per dose administered three times per week during the next course of combined therapy. Presently, this patient is doing well and continuing on with additional rounds of Rexin-G/gemcitabine combined therapy in the hope that the limited oncolytic efficacy of the anti-metabolite may be enhanced by the targeted anti-angiogenic, anti-tumor activity of Rexin-G, which operates with a distinctly different molecular mechanism-of-action.

\section{Discussion}

In a scholarly discussion of an entirely new, vastly different, potentially transformative field of modern medicine, it is important to reflect upon the seminal teachings of Hippocrates, that undisputed paragon and true father of Western medicine, who is credited with advancing the practice of medicine as an honorable profession as well as the systematic study of clinical cause and effect (26). Among the various philosophical considerations regarding patients rights and expectations and the professional obligations and responsibilities of physicians that most concerned Hippocrates, were the vital aspects of avoiding iatrogenic harm, while encouraging continuing education from clinical experience, and a vigilant advancement of the Art, along with the proviso that adequate training in the science is prerequisite. Moreover, in advocating due diligence and humility toward the scientific study of nature: "when Nature leads the way to what is most excellent, instruction in the art takes place', Hippocrates held a particular disdain for those of obstinate authority who continue to remain ignorant with an entrenched and ineffectual apothecary, stating: 'Such persons are like the figures which are introduced in tragedies, for as they have the shape, and dress, and personal appearance of an actor, but are not actors, so also physicians are many in title but very few in reality' (The Law of Hippocrates, circa 400 B.C.).

It is curious, in this day and age, that the global pharmaceutical industry has acquired the assets and intellectual properties of a considerable number of 'gene therapy' companies, but has yet to develop a single gene therapy product of merit; thereby relegating the development and advancement of molecular genetic medicine to academic institutions and affiliated biotechnology companies. In a serious evaluation of the safety and efficacy of our standard treatments for metastatic cancers, in general, and metastatic pancreatic cancer, in particular, it can be concluded that the current state of affairs leaves much to be desired. Not only has precious little been accomplished by the ineffectual apothecaries in terms of improving the survival and the quality-of-life of these metastatic cancer patients - once the initial responses to the metabolic poisons have passed - but the abject failure of chemotherapies to control either the growth or the spread of metastatic disease is one of the most lamentable medical failures of our times $(27,28)$. Worse yet, is the finding that failure to staunch the growth of metastatic pancreatic cancer can often lead to the development of even more dangerous and aggressive forms of the disease, which is concomitant with the development of chemotherapy-resistance $(29,30)$. Likewise, with so-called targeted biologics directed against simplistic receptor-mediated mechanisms: not only is there little or no therapeutic benefit to the pancreatic cancer patient (31-33), but there is the substantial evidence to suggest that there are combined toxicities $(34,35)$, and even rebound in disease progression $(36,37)$, which sheds new light on the issues of comparative safety. 
It is in this context that the timely development of Rexin-G, the world's first and so far only targeted genetic medicine that safely and effectively seeks-out and destroys metastatic cancer should be viewed - in the context of a tragic and historic and yet unmet medical need. It is also important to realize that the unprecedented clinical performance of tumor-targeted genetic medicine is a function of the multiple levels of safety and efficacy embodied in its design engineering (3), as is the observed broad-spectrum anti-cancer activity (16). In terms of safety: i) the stealth vector platform allows repeated infusions without untoward immune responses; ii) the retroviral platform limitation becomes a virtue, as the vector is capable of enforcing gene expression in proliferative/dividing cells only; iii) the growth-associated designer gene is active against cancer cells and proliferative vasculature but not normal non-dividing cells; and iv) the pathotropic accumulation in cancerous tissues essentially sequesters the vector away from non-target organs. In terms of efficacy: i) the cell cycle gene knockout provides for broad-spectrum anti-cancer activity, while ii) the anti-angiogenic activity destroys tumor-associated vasculature, and iii) the pathotropic targeting leads to accumulation and high local concentrations where it is needed most. Moreover, the rational and strategic design engineering of Rexin-G, which is focused on controlling the growthassociated activity of the cyclin G1 proto-oncogene, has received additional scientific validation from recent studies of functional genomics and microRNA action - identifying cyclin G1 blockade as a natural and decisive point of tumor suppression (17) that is apparently more natural and consequential than previously imagined $(18,20)$.

In this report, we provide a compelling glimpse of pathotropic targeting in action, as manifested in a series of noteworthy case studies: cases in which important principles of targeted genetic medicine are brought to bear, and which together, form an advanced practicum for both medical and surgical oncologists. The unique ability of Rexin-G nanoparticles to seek-out, accumulate in, and deliver a lethal payload (cytocidal 'killer gene') to metastatic lesions that have spread throughout the body were clearly shown in two advanced cases of osteosarcoma, the first of which led to the advancement of regulatory approvals from safety to confirmatory efficacy trials, and the second of which led to a complete surgical remission of the previously intractable disease. The need for a refinement of archaic tumor response criteria (i.e., simple RECIST) is further demonstrated in a case of intractable Ewing's sarcoma, and again in a case of intractable breast cancer, where reparative fibrosis and tumorinfiltrating immune responses are seen as beneficial aspects of the patient's own wound healing responses. Such refinement of tumor response criteria is considered to be particularly important in the evaluation of newer targeted therapies, where disease control in the absence of overt toxicity is demonstrated (38). The treatment of late-stage ovarian cancer that had spread to the brain revealed the striking potentiality of Rexin-G to seek out metastatic cancers beyond the bloodbrain barrier, by administering simple intravenous infusions. Among these noteworthy clinical case studies of Rexin-G is the case of the bedridden priest who was suffering from end-stage prostate cancer; for the regression of the primary inoperable mass, the stabilization of extensive bone metastases, the normalization of organ function, and the return to mobility and participation in life in a 91-year-old man, with an inoperable solid tumor, typifies the outstanding safety and single agent-efficacy of Rexin-G in managing yet another type of solid tumor.

Inasmuch as Stage IV pancreatic cancer represents one of the most difficult and deadly forms of cancer, wherein the most advanced of the biologic 'blockbusters' $(32,33)$ and the most extensive arrays of combinatorial chemotherapies $(31,39)$ have failed to reach anything but a nadir in modern medical praxis (28), these noteworthy cases, where Rexin-G at sufficient doses has served to turn the tide of the intractable disease, teaches physicians of the future that it is no longer impossible to manage this dreadful disease. It teaches: i) the advantages of administering Rexin-G as soon as possible in the treatment regimens, ii) the necessity to hold-the-course in the absence of overt toxicity, iii) the empowerment of surgical oncologists with an effective neoadjuvant/adjuvant, who are now able do more for their patients who were previously considered inoperable; it teaches iv) the predictable mechanisms-of-action and v) the revitalization of medicinal pharmacology with the advancement of a singular moleculargenetic medicine and the assertion of a simple treatment formula: [Rexin-G plus $\mathrm{X}$ is greater than $\mathrm{X}$ ] in terms of clinical efficacy and thus improved patient outcome. The quest for synergism with Rexin-G using other agents and antimetabolites is certainly meritorious - now that the overall safety and single-agent efficacy of Rexin-G has been adequately demonstrated in several late-stage cancers. In addition to the obvious first-line combinations of Rexin-G plus $X$, are the possibilities of Rexin- $G$ followed by $X$ regimens, where the clinical evidence for enhanced tumor responsiveness is also mounting.

The number of cancer patients that will benefit from the progressive development of Rexin- $G$ for all solid tumors is expected to increase dramatically in the foreseeable future. Indeed, at the time of this writing, Rexin-G is either registered, conditionally approved, or involved in clinical trials on four separate continents. The attainment of unprecedented tumor control, prolonged survival, and clinical remissions with Rexin-G in an increasing number of late-stage cancer patients is a fitting legacy for all the dedicated scientists and physicians who worked to develop the molecular genetics, to improve upon the core components gene delivery platform, and to increase the biologic potency and effective titers of the clinical grade formulations (3). The progressive development of Rexin-G through the arrays of clinical trials - from prolonged safety studies and compassionate use protocols, to confirmatory trials, expanded access programs, and accelerated approvals, to the advanced dose-dense, neoadjuvent/adjuvant, and combined treatment protocols - is a tribute to the many physicianscientists, clinical investigators, institutional review boards, and conscientious regulatory authorities who, by their diligence and astute guidance, advanced this targeted genetic medicine across the threshold of history. Finally, these monumental clinical advances serve to commemorate the contribution of those courageous cancer patients who participated in these clinical studies with informed and enlightened consent, and in whose bodies the promise and potential of targeted genetic medicine was objectively manifest. For, together with the 
dedicated physicians and scientists, there emerges a vision of the future practice of medicine that outshines the brightest of stars.

\section{Acknowledgements}

The authors would like to acknowledge the contributions of Drs Sant P. Chawla, Jorge G. Ignacio, Roseo E. Manalo, Filomena San Juan, Lionel Baniqued, Alfredo Madamba in these clinical studies; extending our appreciation to Professor Demetrios A. Spandidos, who personally recognized and communicated the biotechnological advancement of Rexin-G through the years, by virtue of the scientific tradition exemplified in his work on cellular oncogenes and the highminded ideals of scholarly communications embodied in his respected medical journals.

\section{References}

1. Retrovector encoding mutant anti-cyclin G1 (Code C49082). NCI thesaurus http://ncit.nci.nih.gov/ncitbrowser/ConceptReport.jsp? dictionary $=\mathrm{NCI} \%$ 20Thesaurus \& code $=\mathrm{C} 49082$

2. Gordon EM and Hall FL: Nanotechnology blooms, at last (Review). Oncol Rep 13: 1003-1007, 2005.

3. Gordon EM, Levy JP, Reed RA, Petchpud WN, Liu L, Wendler CB and Hall FL: Targeting metastatic cancer from the inside: A new generation of targeted gene delivery vectors enables personalized cancer vaccination in situ. Int J Oncol 33: 665-675, 2008

4. Hall FL, Gordon EM, Wu L, Zhu NL, Skotzko MJ, Starnes VA and Anderson WF: Targeting retroviral vectors to vascular lesions by genetic engineering of the MoMuLV gp70 envelope protein. Hum Gene Ther 8: 2183-2192, 1997.

5. Hall FL, Liu L, Zhu NL, Stapfer M, Anderson WF, Beart RW and Gordon EM: Molecular engineering of matrix-targeted retroviral vectors incorporating a surveillance function inherent in von Willebrand factor. Hum Gene Ther 11: 983-993, 2000.

6. Gordon EM, Liu PX, Zhen SH, Liu L, Whitley MD, Gee C, Groshen S, Hinton DR, Beart RW and Hall FL: Inhibition of metastatic tumor growth in nude mice by portal vein infusions of matrix-targeted retroviral vectors bearing a cytocidal cyclin G1 construct. Cancer Res 60: 3343-3347, 2000.

7. Gordon EM, Liu PX, Chen ZH, Liu L, Whitley MD, Liu L, Wei D, Groshen S, Hinton DR, Beart RW, Anderson WF and Hall FL: Systemic administration of a matrix-targeted retroviral vector is efficacious for cancer gene therapy in mice. Hum Gene Ther 12: 193-204, 2001.

8. Wu L, Liu L, Yee A, Carbonaro-Hall D, Tolo V and Hall F: Molecular cloning of the human CYCG1 gene encoding a G-type cyclin: overexpression in osteosarcoma cells. Oncol Rep 1: 705-711, 1994.

9. Xu F, Prescott MF, Liu PX, Chen ZH, Liau G, Gordon EM and Hall FL: Long term inhibition of neointima formation in balloon-injured rat arteries by intraluminal instillation of a matrix-targeted retroviral vector bearing a cytocidal mutant cyclin G1 construct. Int J Mol Med 8: 19-30, 2001.

10. Gordon EM, Chan MT, Geraldino N, Lopez FF, Cornelio GH, Lorenzo CC III, Levy JP, Reed RA, Liu L and Hall FL: Le morte du tumour: histological features of tumor destruction in chemo-resistant cancers following intravenous infusions of pathotropic nanoparticles bearing therapeutic genes. Int J Oncol 30: 1297-1307, 2007.

11. Gordon EM, Cornelio GH, Lorenzo CC, Levy JP, Reed RA, Liu L and Hall FL: First clinical experience using a 'pathotropic' injectable retroviral vector (Rexin-G) as intervention for Stage IV pancreatic cancer. Int J Oncol 24: 177-185, 2004

12. Gordon EM, Lopez FF, Cornelio GH, Lorenzo CC III, Levy JP, Reed RA, Liu L, Bruckner HW and Hall FL: Pathotropic nanoparticles for cancer gene therapy. Rexin- $\mathrm{G}^{\mathrm{TM}}$ : three-year clinical experience. Int J Oncol 29: 1053-1064, 2006.

13. Galanis E, Carlson SK, Foster NR, Lowe V, Quevedo F, McWilliams RR, Grothey A, Jatoi A, Alberts SR and Rubin J: Phase I trial of a pathotropic retroviral vector expressing a cytocidal cyclin G1 construct (Rexin-G) in patients with advanced pancreatic cancer. Mol Ther 16: 979-984, 2008.
14. Chawla SP, Chua VS, Fernandez L, Quon D, Saralou A, Blackwelder WC, Hall FL and Gordon EM: Phase I/II and and Phase II studies of targeted gene delivery in vivo: intravenous Rexin-G for chemotherapy-resistant osteosarcoma and other sarcomas. Mol Ther 17: 1651-1657, 2009.

15. Chawla SP, Chua VS, Fernandez L, Quon D, Blackwelder WC, Gordon EM and Hall FL: Advanced phase I/II studies of targeted gene delivery in vivo: intravenous Rexin-G for gemcitabineresistant metastatic pancreatic cancer. Mol Ther:doi:10.1038/ mt2009.228, 2009.

16. Gordon EM and Hall FL: The 'timely development' of Rexin-G: first targeted injectable gene vector (Review). Int J Oncol 35: 229-238, 2009.

17. Gramantieri L, Ferracin M, Fornari F, Veronese A, Sabbioni S, Liu CG, Giovannini C, Ferrazzi E, Grazi GL, Croce CM, Bolondi L and Negrini M: Cyclin G1 is a target of miR-122a, a microRNA frequently down-regulated in human hepatocellular carcinoma. Cancer Res 67: 6092-6099, 2007.

18. Fornari F, Gramantieri L, Giovannini C, Veronese A, Ferracin M, Sabbioni S, Calin GA, Grazi GL, Croce CM, Tavolari S, Chieco P, Negrini M and Bolondi L: MiR-122/cyclin G1 interaction modulates 53 activity and affects doxorubicin sensitivity of human hepatocarcinoma cells. Cancer Res 69: 5761-5767, 2009.

19. Bai S, Nasser MW, Wang B, Hsu SH, Datta J, Kutay H, Yadav A, Nuovo G, Kumar P and Ghoshal K: MicroRNA-122 inhibits tumorigenic properties of hepatocellular carcinoma cells and sensitizes these cells to sorafenib. J Biol Chem 284: 32015-32027, 2009.

20. Coulouarn C, Factor VM, Andersen JB, Durkin ME and Thorgeirsson SS: Loss of miR-122 expression in liver cancer correlates with suppression of the hepatic phenotype and gain of metastatic properties. Oncogene 28: 3526-3536, 2009.

21. Gordon EM and Hall FL: A primer on pathotropic medicine. In: Celebrating One Hundred Years of the Food and Drug Administration. A Special Centennial Edition. Brooklands New Media Ltd., Oswestry, Shropshire, UK, pp80-83, 2007.

22. Banks WA, Freed EO, Wolf KM, Robinson SM, Franko M and Kumar VB: Transport of human immunodeficiency virus Type 1 pseudoviruses across the blood-brain barrier: role of envelope proteins and adsorptive endocytosis. J Virol 75: 4681-4691, 2001.

23. Groothuis DR: The blood-brain and blood-tumor barriers: a review of strategies for increasing drug concentration. Neurooncology 2: 45-59, 2000.

24. Ko AH, Hwang J, Venook AP, Abbruzzese JL, Bergsland EK and Tempero MA: Serum CA19-9 response as a surrogate for clinical outcome in patients receiving fixed-dose rate gemcitabine for advanced pancreatic cancer. Br J Cancer 93: 195-199, 2005.

25. Wong D, Hwang J, Venook AP, Bergsland EK and Tempero MA: Serum CA19-9 decline compared to radiographic response as a surrogate for clinical outcomes in patients with metastatic pancreatic cancer receiving chemotherapy. Pancreas 37: 269-274, 2008.

26. Hippocrates: The oath and law of Hipprocrates. The Harvard Classics, New York: P.F. Collier \& Son, 1909-14; Bartleby. com, Vol. 38: Part I, 2001. www.bartleby.com/38/1

27. Boeck S and Heinemann V: Second-line therapy in gemcitabinepretreated patients with advanced pancreatic cancer. J Clin Oncol 26: 1178-1179, 2008.

28. Nieto J, Grossbard ML and Kozuch P: Metastatic pancreatic cancer 2008: is the glass less empty? Oncologist 13: 562-576, 2008.

29. Wang Z, Li Y, Kong D, Banerjee S, Ahmad A, Azmi AS, Ali S, Abbruzzese JL, Gallick GE and Sarkar FH: Acquisition of epithelial-mesenchymal transition phenotype of gemcitabineresistant pancreatic cancer cells is linked with activation of the notch signaling pathway. Cancer Res 69: 2400-2407, 2009

30. Arumugam T, Ramachandran V, Fournier KF, Wang H, Marquis L, Abbruzzese JL, Gallick GE, Logsdon CD, McConkey DJ and Choi W: Epithelial to mesenchymal transition contributes to drug resistance in pancreatic cancer. Cancer Res 69: 5820-5828, 2009.

31. Almhanna K and Kim R: Second-line therapy for gemcitabinerefractory pancreatic cancer: is there a standard? Oncology 22: 1176-1183, 2008

32. Burris H III and Rocha-Lima C: New therapeutic directions for advanced pancreatic cancer: targeting the epidermal growth factor and vascular endothelial growth factor pathways. Oncologist 3: 289-298, 2008. 
33. Cartwright T, Richards DA and Boehm KA: Cancer of the pancreas: are we making progress? a review of studies in the US Oncology Research Network. Cancer Control 15: 308-313, 2008.

34. Marshall JL: Vascular endothelial growth factor plus epidermal growth factor receptor dual targeted therapy in metastatic colorectal cancer: synergic or antagonism? J Oncol: 937305 Epub 2009 Dec 6, 2009.

35. Punt CJ and Tol J: More is less - combining targeted therapies in metastatic colorectal cancer. Nat Rev Clin Oncol 6: 731-733, 2009

36. Paez-Ribes M, Allen E, Hudock J, Takeda T, Okuyama H, Vinals F, Inoue M, Bergers G, Hanahan D and Casanovas O: Antiangiogenic therapy elicits malignant progression of tumors to increased local invasion and distant metastasis. Cancer Cell 15: 220-231, 2009
37. Kerbel RS: Issues regarding improving the impact of antiangiogenic drugs for the treatment of breast cancer. Breast 18: S41-S47, 2009.

38. Wahl RL, Jacene H, Kasamon Y and Lodge MA: From RECIST to PERCIST: evolving considerations for PET response criteria in solid tumors. J Nucl Med 50 (Suppl. 1): S122-S150, 2009

39. Roca-Lima CMSP and Flores AM: Gemcitabine doublets in advanced pancreatic cancer: should we move on? J Clin Oncol 24: 327-329, 2006 